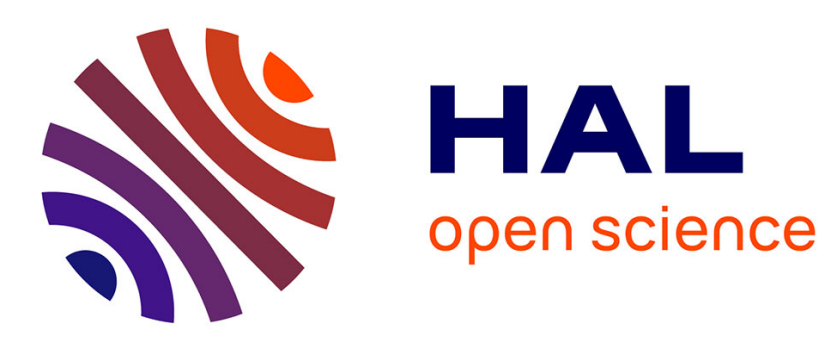

\title{
Étude thermodynamique et expérimentale du système CH4N 2 dans un réacteur à plasma
}

\author{
J. Amouroux, D. Rapakoulias
}

\section{To cite this version:}

J. Amouroux, D. Rapakoulias. Étude thermodynamique et expérimentale du système CH4N

2 dans un réacteur à plasma. Revue de Physique Appliquée, 1977, 12 (7), pp.1013-1021. 10.1051/rphysap:019770012070101300 . jpa-00244270

\section{HAL Id: jpa-00244270 https://hal.science/jpa-00244270}

Submitted on 1 Jan 1977

HAL is a multi-disciplinary open access archive for the deposit and dissemination of scientific research documents, whether they are published or not. The documents may come from teaching and research institutions in France or abroad, or from public or private research centers.
L'archive ouverte pluridisciplinaire HAL, est destinée au dépôt et à la diffusion de documents scientifiques de niveau recherche, publiés ou non, émanant des établissements d'enseignement et de recherche français ou étrangers, des laboratoires publics ou privés. 


\title{
ÉTUDE THERMODYNAMIQUE ET EXPÉRIMENTALE DU SYSTĖME $\mathrm{CH}_{4}-\mathrm{N}_{2}$ DANS UN RÉACTEUR A PLASMA $\left(^{*}\right)$
}

\author{
J. AMOUROUX et D. RAPAKOULIAS
}

Laboratoire de Génie Chimique E. N. S. C. P., 11, rue Pierre et Marie Curie 75231 Paris Cedex 05, France

(Reçu le 30 décembre 1976, accepté le 6 avril 1977).

\begin{abstract}
Résumé. - La mise en œuvre de réacteur industriel au plasma exige de travailler à des pressions suffisamment élevées pour obtenir une production appréciable.

Dans ces conditions il est indispensable de définir les contraintes imposées au système par la thermodynamique dans le cas du système $\mathbf{C}-\mathbf{H}-\mathbf{N}$. L'étude de l'équilibre thermodynamique à haute température permet de justifier le rôle des paramètres de marche des réacteurs de production : composition initiale, température et pression. La température et la composition ont en particulier un rôle déterminant sur la composition du système à l'équilibre. Cette étude précise deux domaines de synthèse chimique l'un correspondant à l'acétylène (vers $1500 \mathrm{~K}$ ) et l'autre à l'acide cyanhydrique (vers $2300 \mathrm{~K}$ ).

Nous avons étudié expérimentalement ces mêmes paramètres dans un réacteur à plasma alimenté par une source haute fréquence de $\mathbf{4 0}$ mégahertz, les résultats obtenus montrent le bon accord avec les prévisions thermodynamiques. Ils soulignent le rôle important joué par l'azote dans le mélange méthane-azote et l'interprétation proposée à partir d'étude bibliographique confirme les mécanismes énoncés.
\end{abstract}

Abstract. - The setting up of industrial plasma chemical reactors with a view to large scale production requires the use of relatively high gas pressures.

Under these conditions it is necessary to define the thermodynamic constraints of the reacting system.

In this paper we have studied the influence of some important working parameters namely : initial composition, pressure and temperature on high-temperature chemical equilibrium of the $\mathrm{C}-\mathrm{H}-\mathrm{N}$ system. The most important parameters are essentially the $N / C$ ratio and the temperature of the system which have a decisive influence upon the mixture composition in chemical equilibrium.

This study shows the presence of two regions for chemical synthesis corresponding first to acetylen synthesis beginning near $1000 \mathrm{~K}$ and decreasing near $2100 \mathrm{~K}$, and second to $\mathrm{HCN}$ synthesis beginning near $1700 \mathrm{~K}$ and decreasing near $2700 \mathrm{~K}$.

We have studied the methane nitrogen mixture in a plasma chemical reactor using a 40 megahertz radiofrequency generator with $3.5 \mathrm{~kW}$ of power. The data are in good agreement with the thermodynamic calculations. This experimental study indicates the nitrogen effect in the $\mathrm{CH}_{4}-\mathrm{C}_{2} \mathrm{H}_{2}$ reaction which can be explained by the transfer between nitrogen and methane as considered by Winckler, Polak and Ricard.

\section{Liste des symboles employés}

$C_{i} \quad$ : Concentration du corps $i$.

$G \quad:$ Enthalpie libre totale du système.

$G_{i} \quad$ : Enthalpie libre molaire du corps $i$.

$\left(G_{T}^{0}\right)_{\mathfrak{f}} \quad:$ Enthalpie libre standard, par mole du corps $i$.

$n \quad:$ Nombre de corps présents à l'équilibre.

$P \quad$ : Pression.

$R \quad$ : Constante des gaz.

$T \quad:$ Température.

$X_{i} \quad$ : Fraction molaire du corps $i$.

$\left(\Delta H_{\mathrm{f}}^{0}\right)_{0}$ : Chaleur standard de formation à $0 \mathrm{~K}$.

$\Delta G_{\mathrm{f}} \quad$ : Enthalpie libre de formation.

(*) Communication présentée au Congrès National de Physique des Plasmas, Paris, 6-10 décembre 1976.
Introduction. - La mise en œuvre de réacteurs chimiques à plasma exige, dans l'optique d'une application industrielle, de définir les variables d'action qui permettront de contrôler les rendements chimique et énergétique du réacteur.

Dans ce but, et quel que soit le type de décharge utilisé, il paraît indispensable de définir les contraintes imposées par la thermodynamique au système à l'équilibre. En effet, cette étude permettra d'une part de justifier du rôle des paramètres : composition du mélange initial, température et pression sur la nature et la composition du mélange à l'équilibre, et d'autre part, d'envisager à partir des espèces radicalaires_et atomiques, les mécanismes d'obtention des espèces-clés recherchées.

Ainsi, elle nous aidera à définir les conditions de 
travail du réacteur et permettra d'identifier les mécanismes de catalyse ou d'inversion des populations des états excités à mettre en œuvre pour modifier le rendement du réacteur.

Dans ce but, nous avons étudié le système $\mathrm{C}-\mathrm{H}-\mathrm{N}$, qui fournit simultanément vers $2000 \mathrm{~K}$ deux espèces d'intérêt industriel : l'acétylène et l'acide cyanhydrique. La première partie de notre travail expose la mise en œuvre de la méthode de calcul, le choix réalisé parmi les espèces chimiques susceptibles d'être présentes à l'équilibre et le rôle des paramètres : composition initiale, pression et température sur la teneur en acétylène et en acide cyanhydrique. La seconde partie présente l'installation expérimentale utilisée et les résultats obtenus. La troisième partie propose une première interprétation des résultats obtenus.

1. Méthode de calcul et mise en œuvre. - L'enthalpie libre d'un système chimique est fonction de la pression, de la température et de la composition du mélange.

$$
G=f\left(P, T, C_{1}, C_{2}, \ldots, C_{n}\right) .
$$

A température et pression constantes, elle peut être calculée à partir de l'enthalpie libre molaire de chaque espèce, $G_{i}$ :

$$
G\left(C_{1}, C_{2}, \ldots, C_{n}\right)=\sum_{i=1}^{n} G_{i} X_{i}
$$

avec

$$
\frac{G_{i}}{R T}=\frac{\left(G_{\mathrm{T}}^{0}\right)_{i}}{R T}+\ln P+\ln \frac{X_{i}}{\sum_{i=1}^{n} X_{i}}
$$

(dans le cas de gaz parfait).

La composition du mélange chimique à l'équilibre est celle qui minimise l'enthalpie libre totale du système, tout en respectant la loi de la conservation de la matière.

La mise en œuvre pratique de la méthode de calcul nécessite la définition des paramètres suivants :

- l'enthalpie libre des espèces chimiques considérées,

- la nature des corps (molécules, radicaux, atomes) susceptibles d'être présents à l'équilibre,

- la composition initiale du mélange,

- la pression et la température.

Le choix des espèces chimiques susceptibles d'être présentes à l'équilibre est basé sur une étude bibliographique préalable $[8,10,11,12,14,15]$.

Après une série de calculs préliminaires, nous avons fixé notre choix sur les 18 espèces suivantes :

- Molécules: $\mathrm{H}_{2}, \mathrm{CH}_{4}, \mathrm{~N}_{2}, \mathrm{C}_{2} \mathrm{H}_{2}, \mathrm{C}_{2} \mathrm{H}_{4}, \mathrm{NH}_{3}$, $\mathrm{C}_{2} \mathrm{H}_{2}, \mathrm{HCN}$.

- Atomes : H, N, C gazeux.

- Radicaux : $\mathrm{CH}, \mathrm{CH}_{2}, \mathrm{CH}_{3}, \mathrm{C}_{2} \mathrm{H}, \mathrm{NH}, \mathrm{NH}_{2}, \mathrm{CN}$.

1.1 ASPECT GÉNÉRAL DES DIAGRAMMES D'ÉQUILIBRE. - Les résultats des calculs permettent de tracer une série de diagramme $X_{i}=f(T)$, représentant la fraction molaire des différents corps à l'équilibre en fonction de la température (Figs 1,2) et pour plusieurs rapports $N / C$. Ils montrent que, sur les 18 espèces chimiques susceptibles d'exister à l'équilibre, 7 d'entre elles n'apparaissent jamais avec une fraction molaire supérieure à $10^{-4}: \mathrm{NH}_{3}, \mathrm{NH}_{2}, \mathrm{NH}, \mathrm{C}_{2} \mathrm{~N}_{2}, \mathrm{CH}_{3}$ et $\mathrm{CH}$.

Parmi les espèces dont la fraction molaire dépasse $10^{-4}$ (et qui seules figurent sur les diagrammes publiés), les principales sont :

- les molécules saturées $\mathrm{CH}_{4}, \mathrm{~N}_{2}$ et $\mathrm{H}_{2}$,

- les molécules $\mathrm{C}_{2} \mathrm{H}_{2}$ et $\mathrm{HCN}$,

- les radicaux $\mathrm{C}_{2} \mathrm{H}$ et $\mathrm{CN}$,

- les atomes $\mathrm{H}$ et $\mathrm{C}$.

L'augmentation de la température provoque la disparition de molécules saturées au profit d'espèces insaturées puis de radicaux et d'atomes.

1.2 INFLUENCE DE LA TEMPÉRATURE. - Une observation des diagrammes d'équilibre permet de distinguer quatre domaines de température, caractérisés par la présence de composés de structure différente.

Entre 500 et $900 \mathrm{~K}$ les seules molécules stables sont $\mathrm{CH}_{4}$ et $\mathrm{N}_{2}$. De 900 à $1300 \mathrm{~K}, \mathrm{CH}_{4}$ se décompose entièrement, tandis que $\mathrm{N}_{2}$ reste stable. L'éthylène a un domaine d'existence étroit et sa concentration reste faible. L'acétylène devient peu à peu l'espèce carbonée majoritaire. Ensuite entre 1300 et $2300 \mathrm{~K}, \mathrm{C}_{2} \mathrm{H}_{2}$ puis $\mathrm{HCN}$ sont les corps carbonés majoritaires accompagnés de $\mathrm{H}_{2}$ et d'une partie de l'azote moléculaire initial. Ce dernier domaine est particulièrement intéressant pour le chimiste, car il correspond à la synthèse de $\mathrm{C}_{2} \mathrm{H}_{2}$ et du $\mathrm{HCN}$.

Enfin, à des températures plus élevées toutes ces molécules se décomposent au profit des radicaux $\mathrm{H}$, $\mathrm{C}_{2} \mathrm{H}, \mathrm{CN}$ et $\mathrm{C}$.

1.3 InfluenCE DU RAPPORT $N / C$. - L'examen des diagrammes montre que la molécule de $\mathrm{HCN}$ apparaît à une concentration significative (fraction molaire supérieure à 0,05 ) pour des rapports $N / C>0,5$. Sa concentration croît régulièrement avec le rapport $N / C$, sauf pour des rapports supérieurs à 10 , où elle décroît. Le comportement de l'acétylène est différent : à basse température $(T<1200 \mathrm{~K})$, l'augmentation du rapport $N / C$ augmente sa stabilité, tandis qu'à des températures plus élevées l'augmentation du rapport $N / C$ lui est fortement défavorable (Fig. 3).

1.4 INFLUENCE CONJUGUÉE DE LA TEMPÉRATURE ET DU RAPPORT $N / C$. - Pour illustrer cette influence nous avons tracé trois diagrammes ternaires. Le premier d'entre eux (Fig. $3 a$ ) correspond à la région de 900 et $1500 \mathrm{~K}$. Les trois espèces carbonées majoritaires dans cette région sont le méthane, l'acétylène et l'acide cyanhydrique.

Pour un rapport $\mathrm{N} / \mathrm{C}$ donné $\mathrm{CH}_{4}$ disparaît lorsque la température augmente, au profit de la formation de 


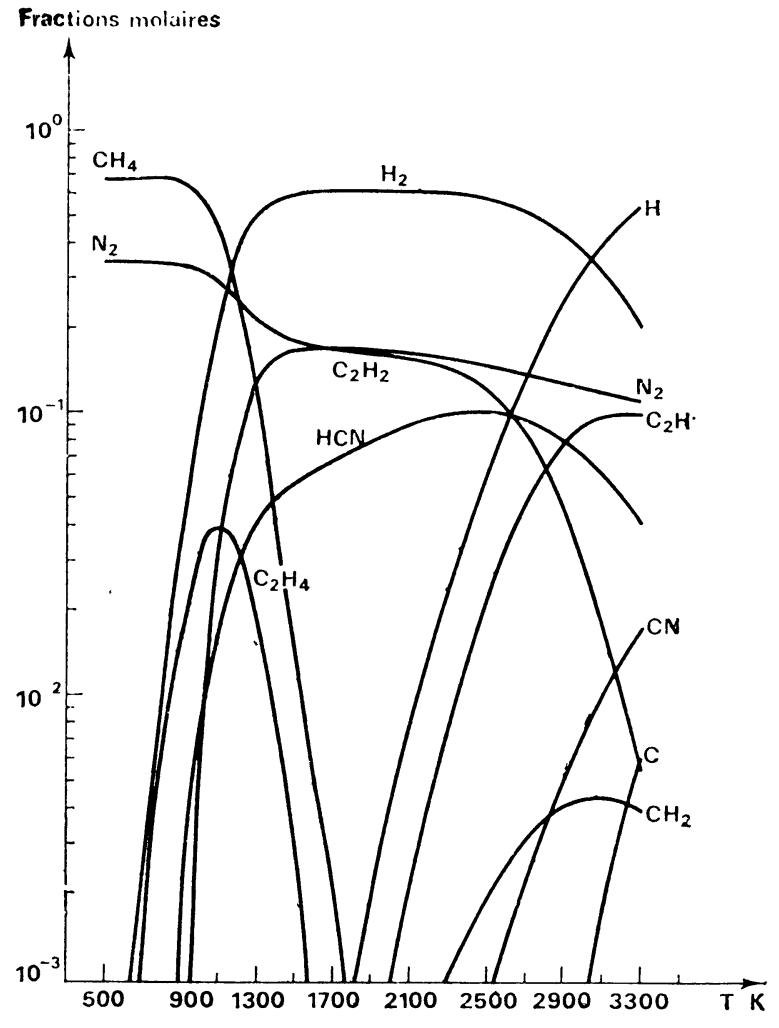

FIG. 1.

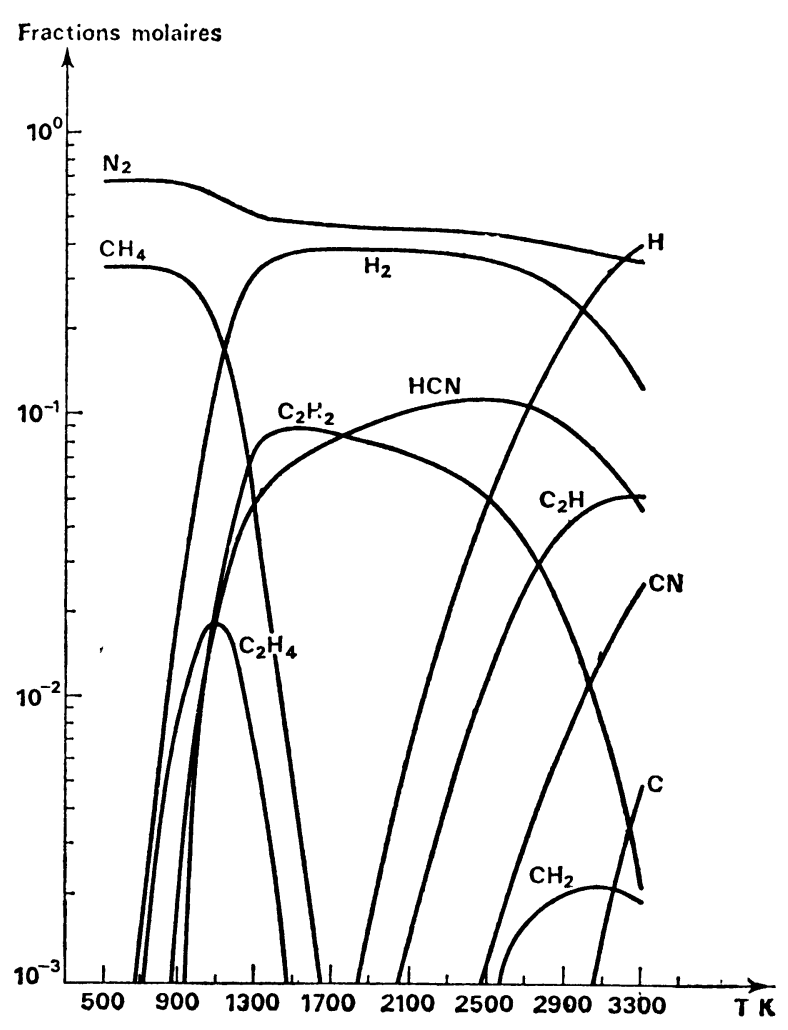

FIG. 2.

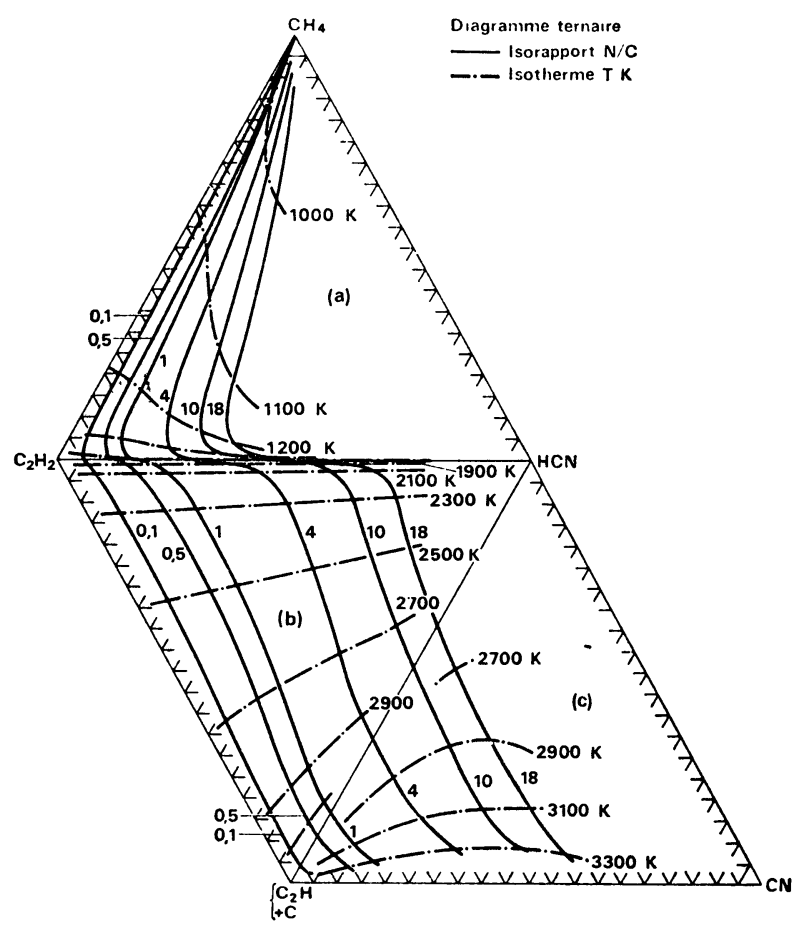

Fig. 3.

Figs. 1, 2, 3. - Diagrammes d'équilibres.

[Equilibrium diagrams.] 
$\mathrm{C}_{2} \mathrm{H}_{2}$ et de $\mathrm{HCN}$. Cette évolution peut être décrite par les réactions:

$$
\begin{aligned}
2 \mathrm{CH}_{4} & \rightarrow \mathrm{C}_{2} \mathrm{H}_{2}+3 \mathrm{H}_{2} \\
\mathrm{CH}_{4}+\mathrm{N} & \rightarrow \mathrm{HCN}+3 / 2 \mathrm{H}_{2} .
\end{aligned}
$$

Cette dernière réaction permet donc de mesurer la teneur en azote dissocié dans le réacteur à plasma.

Pour une température donnée, l'augmentation du rapport $N / C$ favorise $\mathrm{HCN}$, sans pour autant défavoriser $\mathrm{C}_{2} \mathrm{H}_{2}$ (sauf pour des températures inférieures a $1200 \mathrm{~K})$. Dans ce domaine de température, l'acétylène est particulièrement stable, et il se forme préférentiellement à $\mathrm{HCN}$, ce qui explique la zone interdite située à la moitié droite du diagramme ternaire.

Le deuxième diagramme ternaire (Fig. $3 b$ ), correspond à la région de 1500 à $2700 \mathrm{~K}$. Les trois espèces majoritaires sont les molécules $\mathrm{C}_{2} \mathrm{H}_{2}$ et $\mathrm{HCN}$ et le radical $\mathrm{C}_{2} \mathrm{H}$. Pour un rapport $N / C$ donné, $\mathrm{C}_{2} \mathrm{H}_{2}$ disparaît lorsque la température augmente au profit du radical $\mathrm{C}_{2} \mathrm{H}$, alors que la teneur de $\mathrm{HCN}$ reste inchangée. Cette évolution est due à la réaction élémentaire :

$$
\mathrm{C}_{2} \mathrm{H}_{2} \rightarrow \mathrm{C}_{2} \mathrm{H}+\mathrm{H} \text {. }
$$

Pour une température donnée, l'accroissement du rapport $N / C$ favorise $\mathrm{HCN}$ au détriment de $\mathrm{C}_{2} \mathrm{H}_{2}$, selon la réaction élémentaire :

$$
\mathrm{C}_{2} \mathrm{H}_{2}+2 \mathrm{~N} \rightarrow 2 \mathrm{HCN} \text {. }
$$

Le troisième diagramme ternaire (Fig. 3c) décrit l'évolution du système dans la région de température

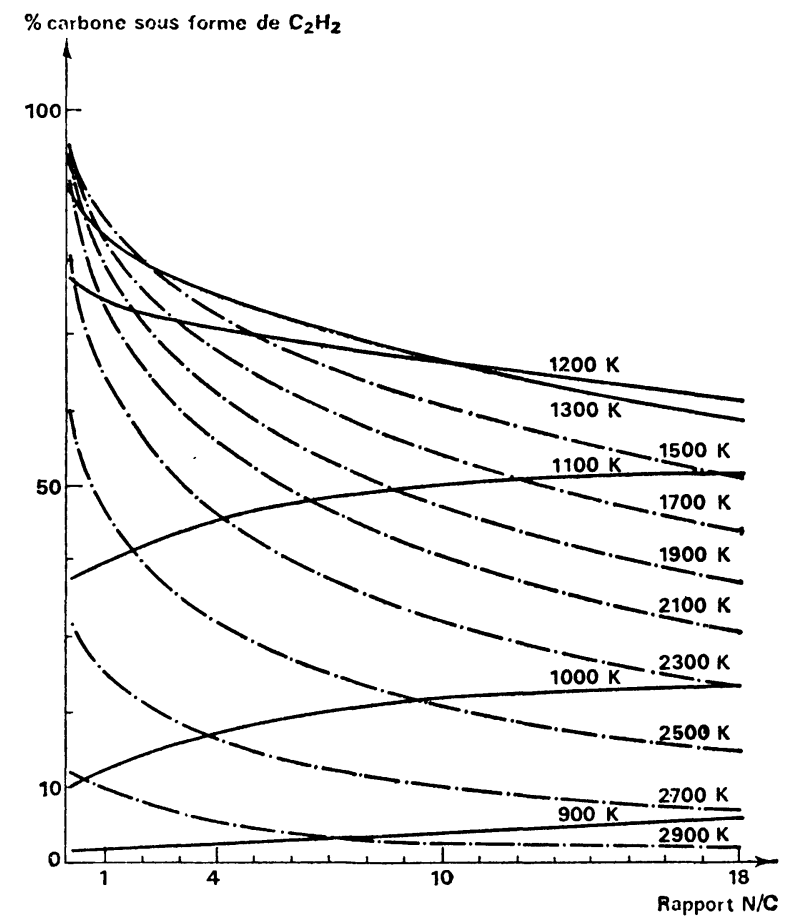

Fig. 4. - Taux de carbone sous forme d'acétylène en fonction du rapport $N / C$ et de la température optimale $P=0,01 \mathrm{~atm}$. $C / H=0,250$.

[Acetylene rate as a function of $N / C$ ratio for different temperatures : $P=0.01 \mathrm{~atm} ; C / H=0.250$.] comprise entre 2700 et $3300 \mathrm{~K}$. Les trois espèces majoritaires sont désormais la molécule $\mathrm{HCN}$ et les deux radicaux $\mathrm{C}_{2} \mathrm{H}$ et $\mathrm{CN}$. Vers $2900 \mathrm{~K}$ la teneur de $\mathrm{HCN}$ décroît et se rapproche de celle du radical $\mathrm{CN}$. De même, la teneur de l'atome $C$ est voisine de celle du radical $\mathrm{C}_{2} \mathrm{H}$.

Pour un rapport $N / C$ donné, la concentration de HCN décroît lorsque la température augmente, au profit du radical $\mathrm{CN}$, alors que celle des deux espèces $\mathrm{C}_{2} \mathrm{H}$ et $\mathrm{C}$ ne varie pas sensiblement. Le changement principal dans ce domaine peut donc être schématisé par la réaction suivante :

$$
\mathrm{HCN} \rightarrow \mathrm{CN}+\mathrm{H} .
$$

Pour une température donnée, lorsque le rapport $N / C$ croit, la concentration du radical $\mathrm{CN}$ augmente, alors que celle des deux radicaux $\mathrm{C}_{2} \mathrm{H}$ et $\mathrm{C}$ diminue, celle de $\mathrm{HCN}$ restant essentiellement constante.

Cette évolution est représentée par les réactions :

$$
\begin{aligned}
\mathrm{C}_{2} \mathrm{H}+2 \mathrm{~N} & \rightarrow 2 \mathrm{CN}+2 \mathrm{H} \\
\mathrm{C}+\mathrm{N} & \rightarrow \mathrm{CN} .
\end{aligned}
$$

1.5 INFLUENCE DE LA PRESSION. - Nous avons tracé les diagrammes $X_{i}=f(T)$ pour une pression totale de 0,1 atm et 0,01 atm (Fig. 5).

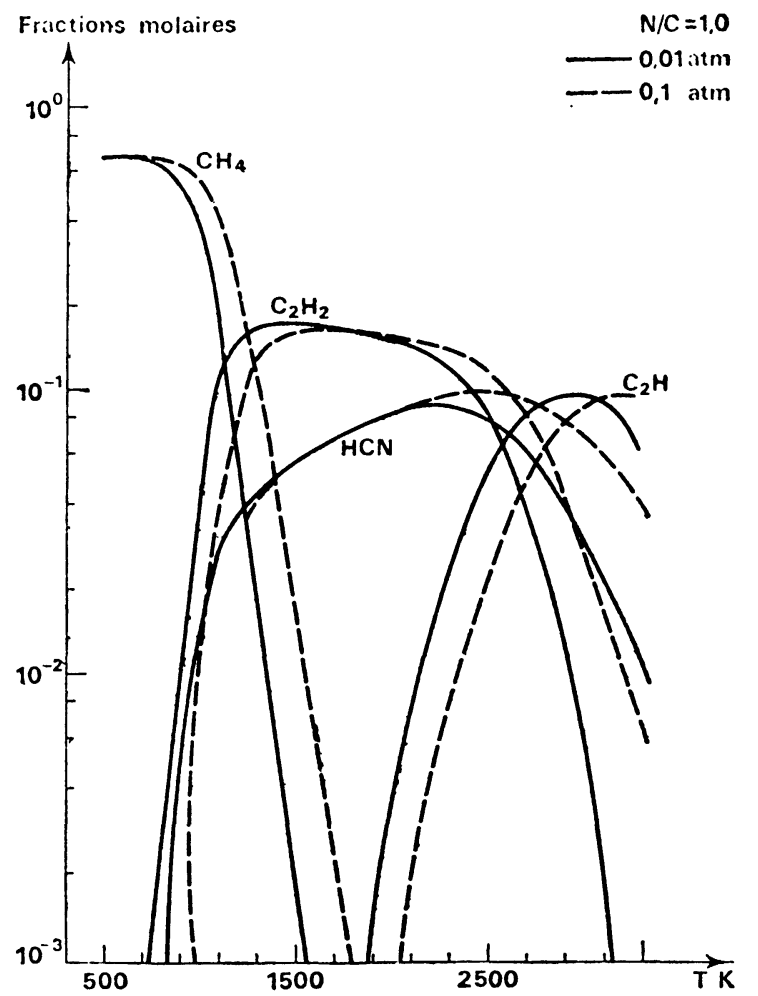

FIG. 5. - Influence de la pression sur la concentration des espèces carbonées majoritaires, pour $N / C=1,0$.

[Pressure effect on carbon species rate for $N / C=1.0$.]

La comparaison de ces diagrammes permet de conclure que l'augmentation de pression de 0,01 à $0,1 \mathrm{~atm}$ ne modifie pas sensiblement la répartition des corps à l'équilibre. 
Un déplacement des courbes d'équilibre vers des températures plus élevées est toutefois observé. Ainsi la température optimale d'apparition de l'acétylène passe de 1300 à $1500 \mathrm{~K}$ et celle de $\mathrm{HCN}$ de 2200 à $1500 \mathrm{~K}$.

La teneur en azote la plus favorable à la synthèse de HCN n'est pas modifiée par l'élévation de la pression. On observe seulement une légère augmentation de la concentration maximale de $\mathrm{HCN}$ qui passe de 10 à $11 \%$ (en moles).

2. Caractéristiques de l'installation. - L'installation se compose de deux parties: d'une part le réacteur chimique et ses annexes de fonctionnement : le générateur haute fréquence et le groupe de pompage : d'autre part les appareils de mesure (Fig. 6).

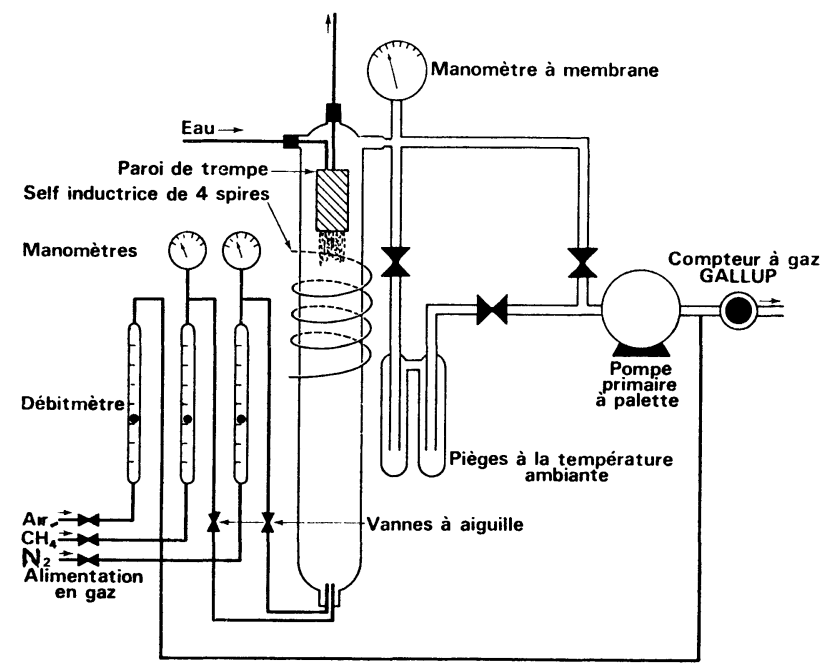

FIG. 6. - Schéma d'installation.

[Experimental apparatus.]

2.1 Le GÉNÉRATEUR HAUTE FRÉQUeNCE. - C'est un oscillateur radio fonctionnant à la fréquence imposée de $38 \mathrm{MHz}$ et délivrant une puissance de sortie maximum de $3,5 \mathrm{~kW}$, sous tension variable de 0 à $5000 \mathrm{~V}$. Couplée au réacteur par une self constituée par 4 spires de cuivre, concentrique au tube de décharge.

2.2 Le RÉACTEUR CHIMiQue. - Il se compose d'un tube de verre du diamètre intérieur de $100 \mathrm{~mm}$ et de longueur $1000 \mathrm{~mm}$ disposé verticalement. Il admet à sa base par deux conduites indépendantes, le méthane et l'azote ; après s'être mélangés dans le tronçon inférieur les gaz entrent dans la zone réactionnelle constituée par le plasma. Cette zone s'étend de la partie supérieure de la self jusqu'à la paroi de trempe constituée par un cylindre de nickel refroidi par une circulation d'eau : comme ce cylindre est relié à la masse électrique, le plasma tend à s'y accrocher, favorisant ainsi les processus de trempe.

3. Résultats expérimentaux. - Les paramètres qui contrôlent le fonctionnement d'un réacteur à plasma sont l'énergie induite dans le mélange, la composition initiale du mélange gazeux, la pression et le temps de passage ainsi que la vitesse de trempe et ses conditions de mise en œuvre. Au cours de nos essais nous avons étudié successivement chacun d'entre eux, en conservant toutefois le même dispositif de trempe.

3.1 INFLUENCE DE L'ÉNERGIE INDUITE. - Ce paramètre représente la quantité d'énergie fournie par le générateur au niveau de la bobine inductrice. Afin de rendre les essais comparatifs, nous l'avons rapporté à l'unité de débit de gaz introduit dans le réacteur. Compte tenu des mesures de température effectuées par thermocouple, nous pouvons dire qu'il existe une corrélation entre l'augmentation de l'énergie induite et la température des neutres.

3.1.1 Variation de la teneur en méthane et en acétylène en sortie de réacteur. - L'introduction d'azote dans le réacteur accélère le processus de pyrolyse du méthane pour une énergie induite donnée. Pour un taux de décomposition de $50 \%$ l'introduction d'azote dans le rapport $N / C=1,25$ diminue de $25 \%$ l'énergie nécessaire à la transformation. Parallèlement l'acétylène, principal produit de synthèse apparaît aux énergies induites faibles, sa teneur maximale dépend par contre du rapport $N / C$ (Figs. 7, 8, 9).

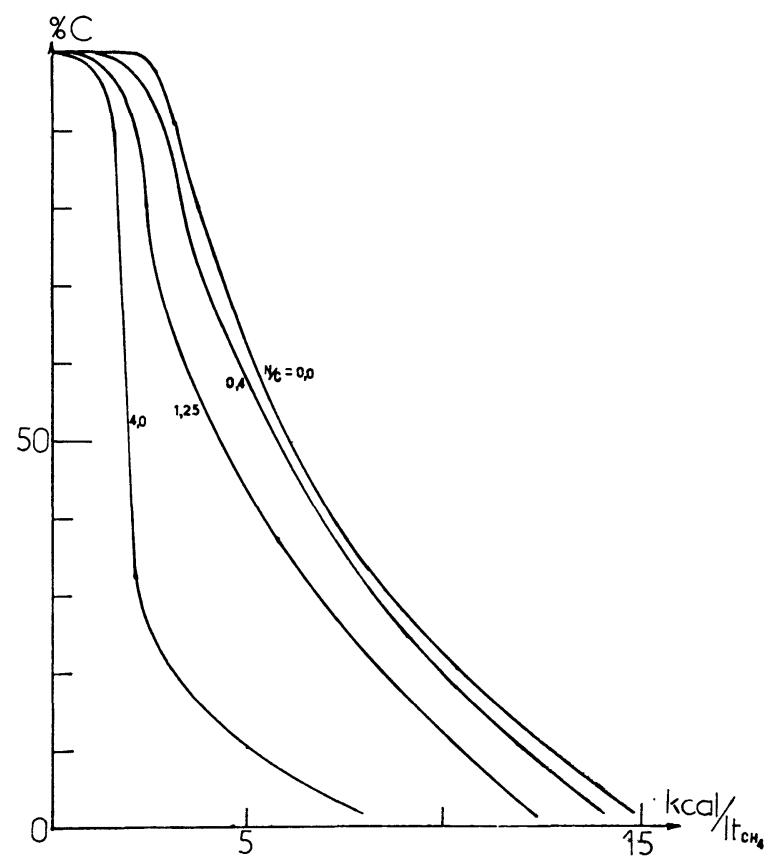

Fig. 7. - Pourcentage du carbone total resté sous forme de $\mathrm{CH}_{4}$ (après la réaction) en fonction de l'énergie induite exprimée en $\mathrm{kcal} / 1$ de $\mathrm{CH}_{4}$.

[Méthane rate at the out-put as a function of induced energy (kcal/1 $\left.\mathrm{CH}_{4}\right)$.]

Ces résultats confirment une partie des conclusions de la thermodynamique quant au déplacement du domaine de formation de l'acétylène vers des températures plus basses lorsque le rapport $N / C$ augmente. 


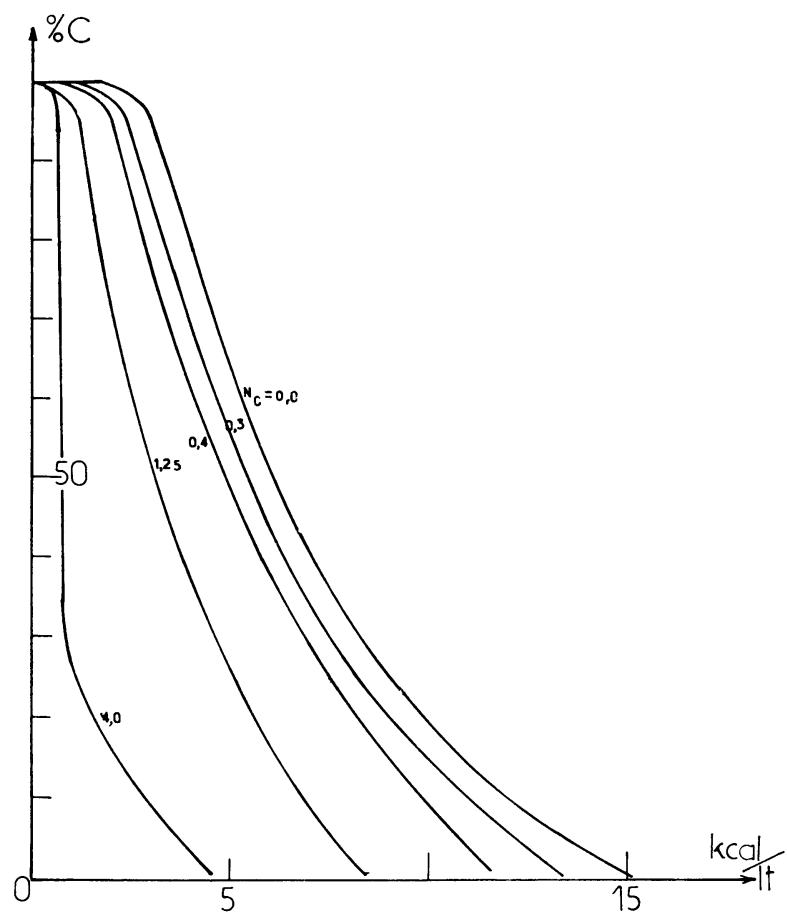

Fig. 8. - Pourcentage du carbone total resté sous forme de $\mathrm{CH}_{4}$ après la réaction, en fonction de l'énergie induite exprimée kcal/l de gaz. Pression 10 torrs.

[Methane rate at the output as a function of the induced energy (kcal/l de gaz) $P=10$ torrs.]

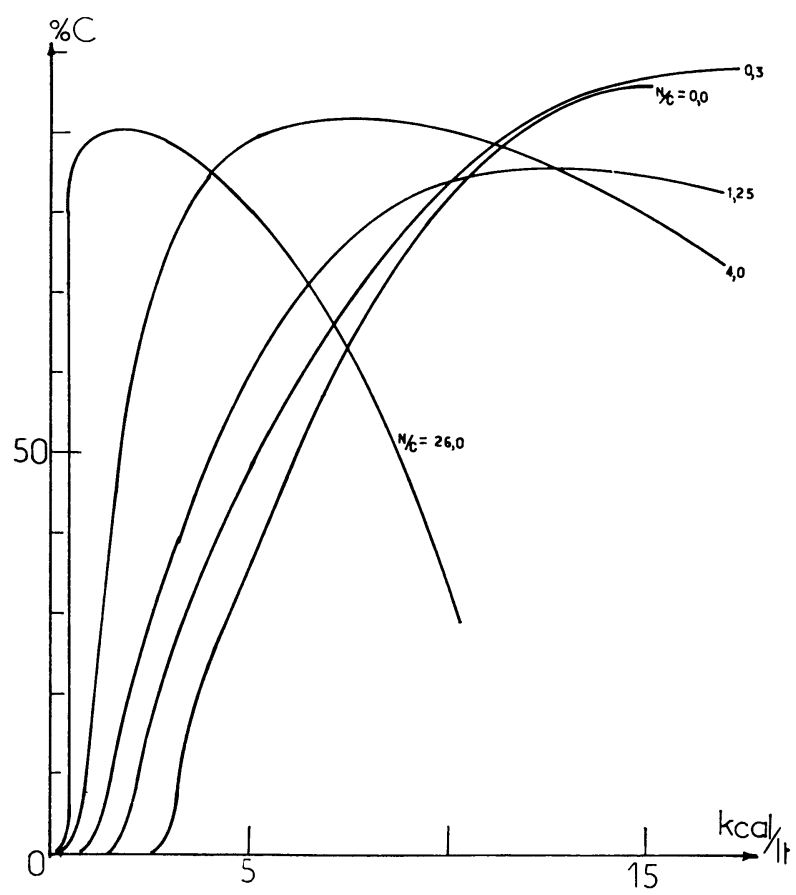

Fig. 9. - Pourcentage du carbone total transformé en $\mathrm{C}_{2} \mathrm{H}_{2}$ en fonction de l'énergie induite. Pression 10 torrs.

[Acetylene rate as a function of the induced energy. $P=10$ torrs.]

3.1.2 Variation de la teneur en acide cyanhydrique. - Le taux de conversion du carbone en acide cyanhydrique augmente avec l'énergie induite, toutefois sa valeur reste faible pour les conditions de fonctionnement, indiquant que la teneur en azote dissociée doit être faible (Fig. 10).

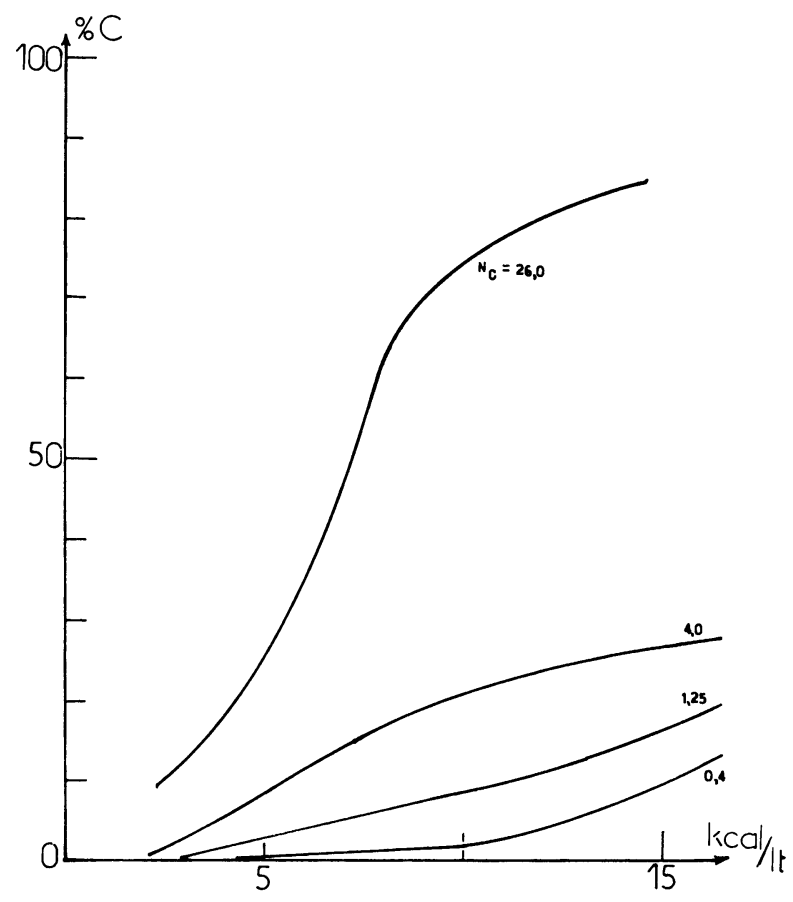

FIG. 10. - Pourcentage du carbone total transformé en $\mathrm{HCN}$ en fonction de l'énergie induite. Pression 10 torrs.

[HCN rate as a function of the induced energie $P=10$ torrs.]

Comme nous l'avons expliqué la synthèse d'acétylène et d'acide cyanhydrique correspond à 2 mécanismes compétitifs dont la sélectivité dépend de la température de travail.

3.2 INFLUENCE DU RAPPORT N/C. - L'influence de la teneur en azote sur les produits a été étudiée pour des rapports compris entre $N / C=0$ et $N / C=26$. Cette étude a été réalisée pour des pressions de travail indiqué et pour différentes énergies induites.

La figure 11, présente la variation respective d'acéty-

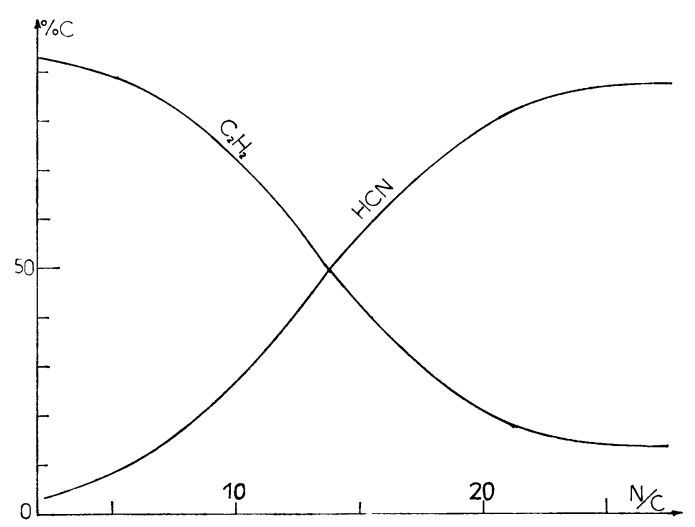

Fig. 11. - Pourcentage du carbonne total transformé en $\mathrm{C}_{2} \mathrm{H}_{2}$ et $\mathrm{HCN}$ en fonction du rapport $N / C$. Pression de travail 20 torrs. Energie induite $15,5 \mathrm{kcal} / \mathrm{l}$ de $\mathrm{gaz}$.

[Percentage of carbon transformed into $\mathrm{C}_{2} \mathrm{H}_{2}$ and $\mathrm{HCN}$ as a function of $N / C$ ratio $P=20$ torrs ; $E$ induced $=15.5 \mathrm{kcal} / 1$.] 
lène et d'acide cyanhydrique en fonction du rapport $N / C$ et pour une même énergie induite par litre de gaz. Aux faibles rapports $N / C$ l'acétylène est l'espèce majoritaire, et l'acide cyanhydrique apparaît au détriment de l'acétylène lorsque la teneur en azote augmente.

Cette étude a été effectuée pour différentes énergies induites et la figure 12 montre que la teneur en acéty-

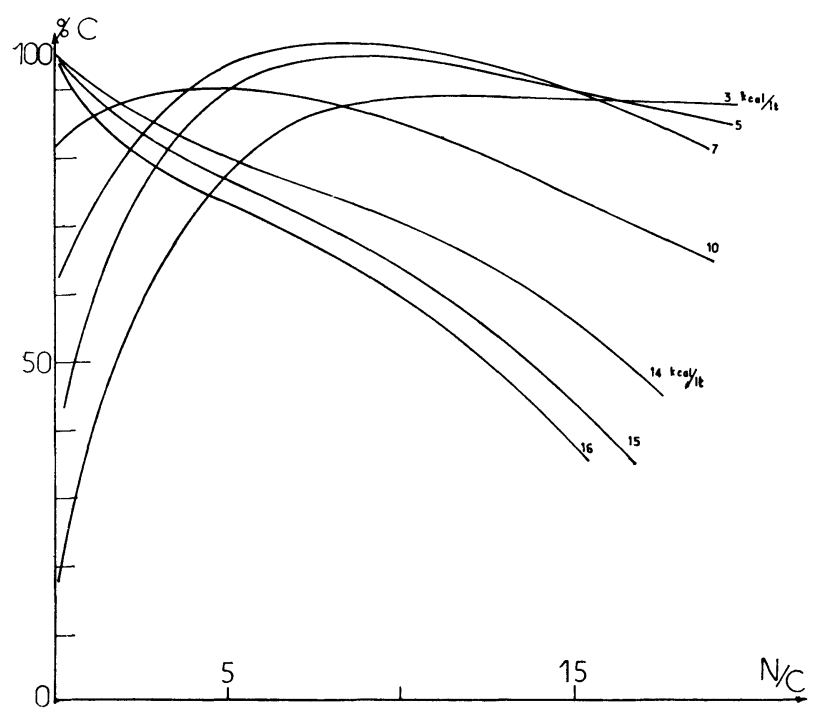

FIG. 12. - Pourcentage de carbone transformé en $\mathrm{C}_{2} \mathrm{H}_{2}$ en fonction du rapport $N / C$. Pression 10 torrs.

[Acetylene as a function of $N / C$ ratio ; $P=10$ torrs.]

lène augmente avec le rapport $N / C$ aux faibles énergies induites, par contre elle décroît avec le rapport $N / C$ aux fortes énergies induites. Ce travail confirme l'étude thermodynamique qui définit les conditions de formation de l'acétylène en fonction du rapport $N / C$ et pour différentes températures.

Enfin nous constatons (Fig. 13) que la teneur en azote fixée sous forme de $\mathrm{HCN}$ en sortie du réacteur est constante quel que soit le rapport $N / C$. Le réacteur fonctionnait alors à une pression de 20 torrs et l'énergie induite était de $15 \mathrm{kcal} / \mathrm{l}$ de gaz. Cette conclusion

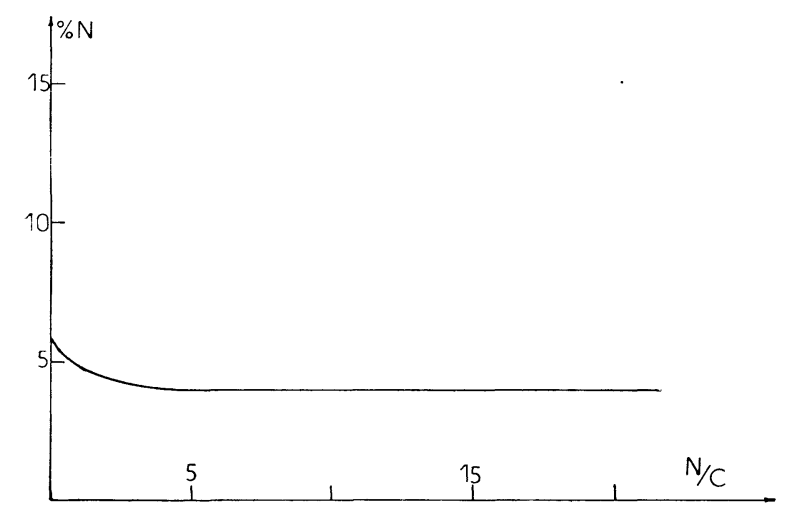

FIG. 13. - Pourcentage d'azote total transformé en $\mathrm{HCN}$ en fonction du rapport $N / C$.

[Nitrogen fixed (HCN) as a function of $N / C$ ratio.]

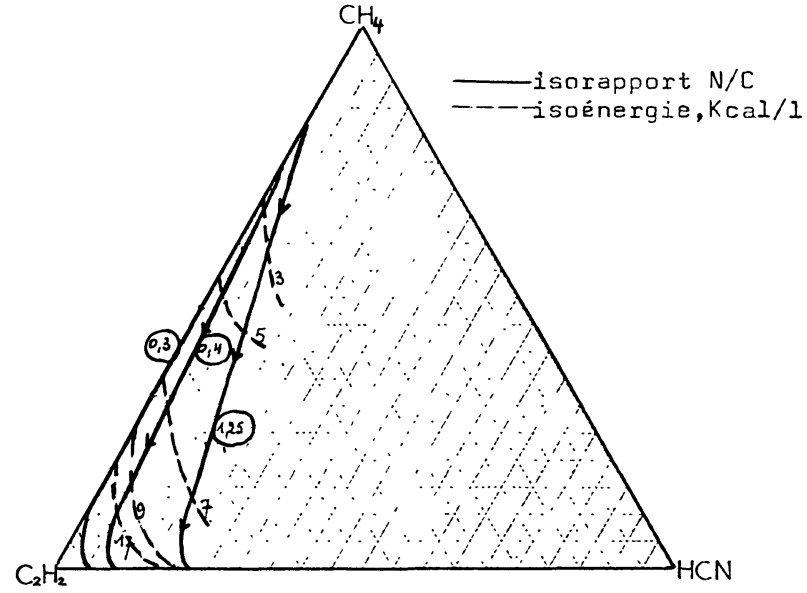

FIG. 14. - Diagramme ternaire expérimental. Pression 20 torrs. [Experimental ternary diagram ; $P=20$ torrs.]

indique que le taux d'azote fixé est constant quel que soit le débit d'azote introduit.

3.3 INFLUENCE DE LA PRESSION. - L'augmentation de la pression de travail du réacteur (Fig. 15) (10 à 20 torrs) favorise l'apparition de l'acide cyanhydrique

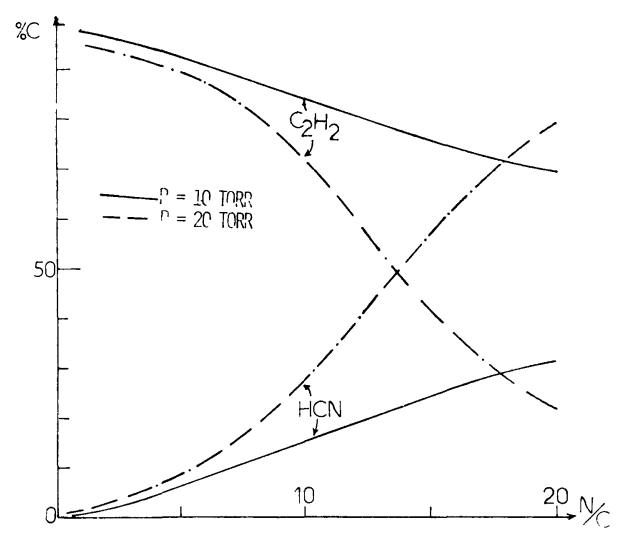

FIG. 15. - Influence de la pression sur le taux de transformation du $\mathrm{CH}_{4}$ en $\mathrm{C}_{2} \mathrm{H}_{2}$ et en $\mathrm{HCN}$, en fonction du rapport $N / C$. Energie $15,5 \mathrm{kcal} / \mathrm{l}$ de gaz.

[Pressure effect on $\mathrm{C}_{2} \mathrm{H}_{2}$ and $\mathrm{HCN}$ rate produced as a function of $N / C$ ratio $(E$ induced $15.5 \mathrm{kcal} / \mathrm{l})$.]

au détriment de l'acétylène. Cette conclusion doit être attribuée à l'élévation de la température des neutres avec la pression. En effet l'augmentation de pression s'accentue dans la zone réactionnelle par suite de l'effet de pincement du plasma. Dans ces conditions les chocs thermiques augmentent, favorisant l'apparition de l'acide cyanhydrique dont le domaine de formation se situe vers $2300 \mathrm{~K}$.

3.4. Influence du temps de passage. - Ce paramètre représente le rapport débit/volume pour un réacteur, il détermine la taille de l'installation nécessaire à une production. Pour l'étudier, nous avons fait varier la longueur de la zone réactionnelle comprise 
entre la self inductrice et la paroi de trempe. Nous constatons à partir de la figure 16 que le temps de passage n'est pas un paramètre critique ce qui suppose que le réacteur a atteint un état d'équilibre. Cette

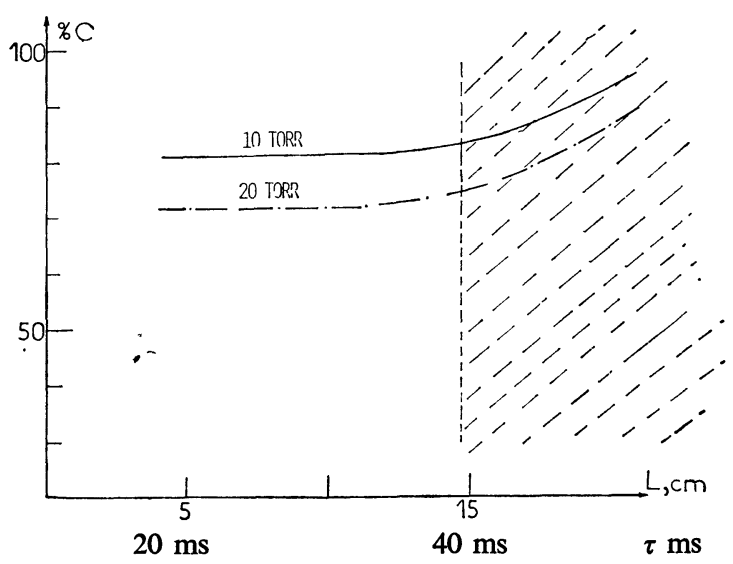

Fig. 16. - Influence du temps de passage sur le taux de conversion de carbone en acétylène.

[Influence of the time of passage on the conversion rate carbonacetylene.]

conclusion est particulièrement importante puisqu'elle justifie avec les autres observations le bon accord entre la thermodynamique et les résultats expérimentaux. Enfin le diagramme ternaire (Fig. 14) tracé à partir des données expérimentales est en accord avec celui obtenu avec l'étude thermodynamique.

4. Interprétation des résultats. - Les principales conclusions qui apparaissent à partir des essais effectués s'énoncent de la façon suivante :

1) la présence d'azote facilite la décomposition du méthane ;

2) le taux d'acétylène formé passe par un maximum en fonction de l'énergie induite ;

3) le taux d'acide cyanhydrique crôit au détriment de l'acétylène ;

4) le taux d'azote fixé est indépendant du rapport $N / C$;

5) la variation du temps de passage ne modifie pas le rendement ce qui signifie que l'équilibre est atteint.

Pour interpréter ces résultats il est nécessaire de rappeler les caractéristiques respectives des deux molécules de base : $\mathrm{CH}_{4}$ et $\mathrm{N}_{2}$.

Le tableau (I) permet de présenter les différences

\section{TABleaU I}

$\begin{array}{lcc} & N_{2} & \mathrm{CH}_{4} \\ \text { Dissociation } & \overline{-} & \overline{-} \\ & 9,76 \mathrm{eV} & 4,53 \mathrm{eV} \\ \text { Excitation } & 6,17 \mathrm{eV} & \left(\mathrm{CH}_{3}+\mathrm{H}\right) \\ & 7,32 \mathrm{eV} & 10,12 \mathrm{eV} \\ & 11,88 \mathrm{eV} & 11,8 \mathrm{eV} \\ \text { Ionisation } & 15 \mathrm{eV} & 13,27 \mathrm{eV}\end{array}$

entre ces deux espèces du point de vue de leur dissociation, excitation et ionisation.

La décharge HF employée est caractérisée par une énergie électronique moyenne comprise entre 1 et $2 \mathrm{eV}$ (si l'on utilise l'expression de Von Engel). Dans ces conditions la fraction de puissance transférée le sera sous forme vibrationnelle et électronique (Fig. 17) le processus de transfert par ionisation étant négligeable.

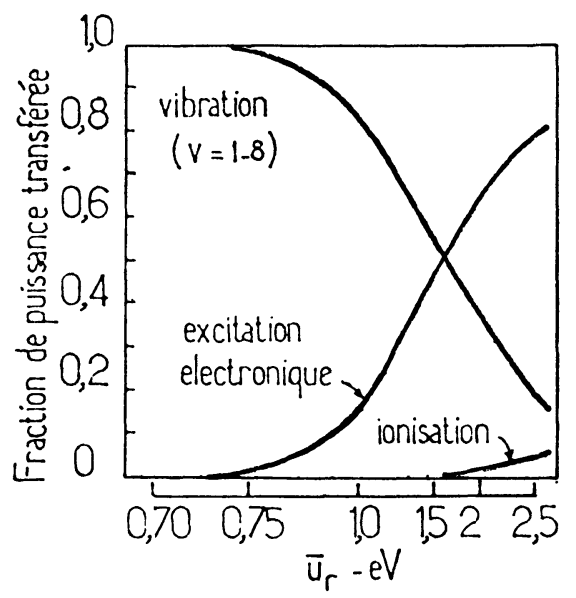

Fig. 17. - Transfert aux molécules par collisions électroniques dans une décharge d'azote (d'après [14]).

[Energy transfer to the molecules by electronic collisions in a nitrogen discharge.]

Or l'azote $(6,17 \mathrm{eV})$ ayant un potentiel d'excitation plus faible que le méthane $(8 \mathrm{eV})$ absorbe plus facilement l'énergie, par contre sa dissociation $(9,7 \mathrm{eV})$ sera plus difficile que celle du méthane $(4,5 \mathrm{eV})$. Ainsi le transfert d'énergie entre l'azote et le méthane semble justifier l'amélioration du processus de pyrolyse observé lorsque l'on introduit de l'azote dans le réacteur. Winckler a montré expérimentalement que la principale réaction de décomposition du méthane dans un mélange $\mathrm{CH}_{4}-\mathrm{N}_{2}$ est :

$$
\begin{aligned}
\mathrm{CH}_{4}+\mathrm{N}_{2}\left(\mathrm{~A}^{3} \Sigma \mathrm{u}^{+}\right) \rightarrow \underset{\mathrm{CH}_{4}^{*}}{{ }^{*}}+\mathrm{N}_{2}\left(\mathrm{X}, \Sigma \mathrm{g}^{+}\right) \\
\longrightarrow \mathrm{CH}_{3}+\mathrm{H}
\end{aligned}
$$

ce mécanisme confirme les résultats expérimentaux observés.

La formation de l'acide cyanhydrique exige la présence d'azote dissocié mais sa teneur est faible à la sortie du réacteur. En effet compte tenu de l'énergie nécessaire pour obtenir sa dissociation $(10 \mathrm{eV})$, l'apparition d'azote atomique proviendrait d'après Polak, Bell et Ricard de l'excitation vibrationnelle du niveau fondamental dont la durée de vie permet une accumulation par collisions successives de l'énergie nécessaire à la dissociation de la molécule d'azote. Ce mécanisme est important car il permet l'élimination des dépôts de carbone qui accompagne en général la pyrolyse du méthane. 
5. Conclusion. - L'étude théorique et expérimentale du système $\mathrm{CH}_{4}-\mathrm{N}_{2}$ montre dans un premier temps un bon accord entre les prévisions et les résultats de l'expérience. Toutefois la connaissance des mécanismes d'excitation respectifs des molécules $\mathrm{CH}_{4}$ et $\mathrm{N}_{2}$ permet d'expliquer un certain nombre de faits précis observés
(\% d'azote fixé, amélioration du taux de conversion dû à l'azote) et les explications fournies par la bibliographie corroborent les faits expérimentaux. Nous espérons poursuivre ce travail par une étude spectroscopique qui devrait permettre de vérifier les hypothèses énoncées.

\section{Bibliographie}

[1] Alexandrov, N. K., Ganz, S. N., Parkhomenko, V. D., Galoborod'KI, V. P., Zh. Prikl. Khim 46 (1973) 1256.

[2] Amouroux, J., Foll, J. P., Rapakoulias, D., Ann. Chim. 9 (1974) 181.

[3] Capezzuto, P., Cramazossa, F., Ferrato, G., Maione, P., Molinari, E., Gaz. Chim. Ital. 103 (1973) 1153.

[4] Duff, R. E., Bauer, S. H., J. Chem. Phys. 36 (1962) 1754.

[5] Ganz, S. N., Parkhomenko, V. D., Krasnokyt'skiI, V. I., PolyaChIKov, O. I., Izv. vyssh. Ucheb. Zaved, Khim. Khim. Tekhnol. 16 (1973) 741.

[6] Freeman, M. P., Mentzer, C. C., I et EC, Proc.Des. Dev., 9 (1970) 39.

[7] JANAF, Tables of thermochemical data, 1964.

[8] Krasnokyt'skiI, Y. I., GanZ, S. N., Parkhomenko, V. D., Zh. Prikl. Khim. 46 (1973) 2425.

[9] Marynowski, C. W., Phillips, R. C., Phillips, J. R., Hiester, N. K., I et EC Fund. 1 (1962) 52.

[10] Rapakoulias, D., Amouroux, J., J. Ann. Chim. 8 (1973) 193.

[11] Ronneau, C., VRebosh, J., Von Tiggelen, A., Revue de l'IFP XXIII (1968) 236.
[12] BeLl, A. T., in Techniques and applications of plasma chemistry (Willey), 1974.

[13] Broffin, B., in "Chemical Reactions in Electrical Discharges » (ed. Gould R. F.) 1969, p. 423.

[14] Delcroix, J. L., Matos Ferreira, C., Ricard, A., Atomes et Molécules Métastables dans les gaz ionisés. C. N. R. S. 1975

[15] Fauchais, P., Rev. Int. Hautes Temp. Réfract. 6 (1969) 77.

[16] Freeman, M. P., Skrivan, J. F., AICHE J. 8 (1962) 450.

[17] Leutneur, H. W., Ind. Eng. Chem. Proc., Des. Dev. 2 (1963) 315.

[18] Nighan, W. L., Phys. Rev. A 2 (1970) 1989.

[19] Polak, L. S., Pure Appl. Chem. 39 (1974) 307.

[20] Polak, L. S., in Reactions Under Plasma conditions (éd. Venugopalan) Vol. II, 1970.

[21] Tal'rose, V. L., Karachevtsev, G. V., in Reactions Under Plasma conditions. (Ed. Venugopalan) Vol. II, 1970.

[22] Winkler, C. A., Wright, A. N., Can. J. Chem. 40 (1962) 1291. 